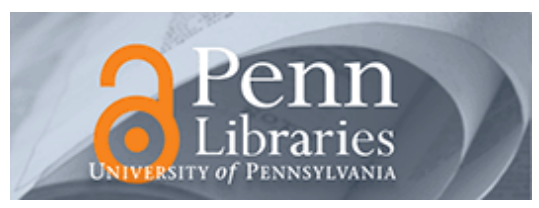

Studies in Visual Communication

Volume 1

Issue 1 Fall 1974

Article 7

1974

\title{
Temporal and Spatial Transitions in American Fiction Films
}

John Carey

\section{Recommended Citation}

Carey, J. (1974). Temporal and Spatial Transitions in American Fiction Films. 1 (1), 45-50. Retrieved from https://repository.upenn.edu/svc/vol1/iss1/7

This paper is posted at ScholarlyCommons. https://repository.upenn.edu/svc/vol1/iss1/7

For more information, please contact repository@pobox.upenn.edu. 


\section{Temporal and Spatial Transitions in American Fiction Films}




\section{TEMPORAL AND SPATIAL TRANSITIONS IN AMERICAN FICTION FILMS}

\section{JOHN CAREY}

This study examines the communication code structure of temporal and spatial transitions in feature length, American fiction films. By these transitions I mean simply, the rules, principles or conventions by which a filmmaker communicates to his audience that the shot or scene they are currently viewing is at a different point in time and/or space than the previous shot or scene. For example, if we are watching a scene that depicts an apartment in New York, and the filmmaker wants to follow this with a scene depicting an apartment in Chicago three days later, how does he communicate this transition to us?

A number of related structural issues will not be treated here. I shall however, mention a few of these briefly, to clarify the scope of my investigation. I am not concerned with the relation between real time and film time. For example, a filmmaker may compress the real time it takes a person to walk across a field by use of a cutaway or change in camera angles. Thus while it takes the actor two minutes to perform the action, the film time for that movement may be fifteen seconds. With rare exception, a filmmaker does not intend to communicate a speeded-up action by his editing of such a movement, and his audience will not infer such a meaning. ${ }^{1}$

Similarly, the stretching or lengthening of real time in a film, for example, Eisenstein's "raising of the bridge" sequence in October, where the bridge seems to rise almost endlessly, or Hitchcock's lengthening of real time in The Lodger, where we see a close shot of the killer switching off the light, followed by a long shot in which the light goes out, are outside the scope of my investigation. In these cases, the filmmaker does indeed attach meaning to his manipulation of real time, but typically, he does not imply a temporal transition. Rather, a viewer will infer "heightened tension," "boredom," or some other feeling from the temporal manipulation.

In addition, I will not consider how a filmmaker constructs temporal and spatial units in a film from elements shot at disparate points in time and space. Pudorkin (1949:88), among others, discusses the process of joining several shots, each filmed in a different place, at a different time, so that a viewer will infer a single, clear, uninterrupted

John Carey is an associate in Environmental Media Consultants where he is currently engaged in research in political communication. He is also working on his doctorate in communication at the Annenberg School of Communications, University of Pennsy/vania. action. This is an important structural issue, but it relates to the construction of film time and space from "real" life, not the communication process between filmmaker and audience. $^{2}$

I am dealing exclusively with the process and conventions whereby a filmmaker intends to convey to his audience that the scene within the ongoing film has shifted in time and space. These conventions may be broadly divided into two groups: single element and multiple element transitions. Single element transitions occur when the previous scene is connected directly to the following scene, with no intermediate shots. For example, we are watching a shot of a room, and the film cuts directly to a shot of a park; or, we are watching a shot of a room and the screen gradually becomes darker, until it is totally black, followed by a gradual lightening of the screen which reveals a new scene in a park (this mechanism is called a fade); or, we are watching a room and the shot of the park gradually dissolves through, replacing the previous shot; or, we are watching a room and the shot of a park starts to move across the screen and seems to push the first shot out of the frame (called a wipe). The cut, fade, dissolve, and wipe are the most common transition mechanisms in the films we will be discussing. The second broad category, multiple element transitions, also use cuts, fades, wipes, and dissolves to link the previous and subsequent scene, but in addition they insert a shot or shots that are part of the transition itself. For example, a scene dissolves through to a shot of a calendar, with pages flipping off a wall, which dissolves through to the next scene; or, a scene in a room dissolves to a long shot of a boat crossing the Atlantic which dissolves to a new scene at another point in time and space.

The study was reduced to this scope in order to deal more clearly with a particular communication problem: how does a filmmaker imply meaning by a structural mechanism in his film, and how does an audience infer meaning? What is the nature of the code they share that allows communication to occur? Bateson (1969) argues that the business of communication is a continuous learning to communicate, and that codes and languages are not static systems which can be learned once and for all, but rather, shifting systems of pacts and premises which govern how messages are to be made and interpreted. Gombrich, an art historian, (1960:370-375) focuses more specifically on visual communication, when he argues that images attain meaning because creator and viewer share a set of conventions by which expressions about visual reality can be coded and decoded. He says an artist discovers "schemata" or a set of conventions known by people at a particular time, in a particular culture, and uses them to create meaning in a visual form. Similarly, Worth (1975:37-40) argues that visual communication takes place not because people are commonly attuned to a universal "reality," but because they have learned the conventions, rules, forms, and structure of a social group. We infer meaning from visual communication not by matching its correspondence to how the world is made but by interpreting it against our knowledge of "how people make pictures, how they made them in the past, how they make them now, and how they will make them for various purposes in various contexts" (Worth 1975:39). I sought to examine these issues within a narrowly defined code. 
The Gombrich-Worth position has not been widely shared among those who have talked about temporal and spatial transitions in film, particularly in the instructional primers on film techniques. Most have treated these mechanisms as a static grammar, an invariant set of rules based on the "innate" properties of visual reality. Arnheim (1957, 1966) provides the theoretical impetus for the commonly held view, with his position that there is an organized world to which we are biologically and perceptually attuned and to which we can respond instantly. Since man is biologically attuned to a "deep visual structure," he does not have to negotiate a system of arbitrary symbolic forms that must be learned by an audience. Rather, a visual stimulus, which has a character of its own and contains objective properties, will steer the organizational properties within the brain and determine the form and meaning of surface structures in a work of art or a film. Thus, the particular use of a fade or dissolve or combination of elements is most frequently considered a surface manifestation of a universal deep visual structure.

There has been little discussion, and less research, about possible variations in code structure diachronically, across cultures, or across film subjects. Some have made judgments about particular transition mechanisms as "more filmic" and therefore good, while other transition mechanisms are judged as weak or uncreative because they are "borrowed" from other modes such as literature, the stage, etc. Balázs, for example, bitterly opposes the wipe as a crude imitation of the stage:

When a director wants a change of scene but does not want to show intermediate scenes, he often has a curtain of shadow, technically termed a "wipe", drawn across the picture. In other words, he begins a new scene by means of a device borrowed from the stage. This admission of impotence, this barbarian bit of laziness, is so contrary to the spirit of film art that the only thing to be said in its defense is that it is nevertheless preferable to a picture cut in without dramaturgical motivation [1970:143] .

Similarly, though with an absence of venom, Arnheim (1957:119) likens the fade to a theater curtain changing scenes in a play.

It has also been suggested that fades, dissolves, and wipes may be the equivalent of linguistic mechanisms. Again, Balázs (1970:143), only now talking about the fade:

Sometimes its effect is like that of a dash in a written text sometimes like a row of full stops after a sentence, leaving it open ....

In addition, various mood feelings have been attributed to these mechanisms. The fade is said to produce sadness; the dissolve, thought-like weightlessness. This suggests that the use of a particular mechanism might correlate with the mood of a scene or the subject of a film.

One can also ask to what degree does the use of a particular temporal-spatial transition mechanism reflect the technological availability of that mechanism to a filmmaker. Goffman (1974:259), talking about the theater, observes that,

The introduction of gaslight in London theaters in 1817 and the introduction of electric spark lighters for gas in the 1850 s made it technically possible to dim and extinguish lights in the auditorium, thereby providing a signal for the beginning and ending of action within the theatrical frame.
While nearly all of the mechanisms used in temporal and spatial transitions were available by the 1920s, we really don't know the shifting costs or work habits of optical houses and production companies over the years. It is interesting to observe the recent increased use of the wipe as a transition mechanism in children's television programming, at a time when much of the editing has shifted to video tape where the wipe is readily available by virtue of editing console design.

We face the possibility that temporal and spatial transition mechanisms may represent: (1) a static system of invariant rules determined by the innate deep structure of visual reality; (2) the visual equivalent of linguistic structure; (3) borrowing from other modes such as theater or literature; (4) technological availability; and/or (5) stylistic variation based on the content of films or the mood of particular scenes.

As a first step in assaying some of these possible explanations, and to place them within a communication framework, I sought to map the temporal-spatial transition mechanisms used by filmmakers diachronically. My sample consisted of three basic categories of fiction films within each decade, ${ }^{3}$ beginning with the 1920s: (1) adventurescience fiction, (2) situation drama, and (3) comedy (the sample was limited to American films). I was concerned primarily with the structural features of a transition-fade or wipe, single element or multiple element transition, time necessary to complete the transition, etc. I also noted semantic features of the transition, e.g., a face dissolving to a flag, insert shots of calendar pages flipping off a wall, or seasons changing, and mood features in scenes where the transition occurs. ${ }^{4}$

The basic pattern shows marked changes in the mechanisms for accomplishing temporal and spatial transitions over time, and yet a consistency in the pattern of using these mechanisms within any period. Filmmakers observe the conventions used by contemporary films, not a set of invariant rules. Further, variations from the code at any point are themselves patterned and accounted for by the code. (See Table I.)

TABLE I

SINGLE ELEMENT TRANSITIONS

\begin{tabular}{lccccc}
\hline & $\begin{array}{c}\text { Fade } \\
* \%(\mathrm{~N})\end{array}$ & Dissolve & Wipe & Cut & Focus \\
\hline $1930 \mathrm{~s}$ & $46(53)$ & $44(50)$ & $9(10)$ & $1(1)$ & $0(0)$ \\
$1940 \mathrm{~s}$ & $27(40)$ & $64(94)$ & $5(8)$ & $3(4)$ & $0(0)$ \\
$1950 \mathrm{~s}$ & $13(18)$ & $66(91)$ & $0(0)$ & $21(29)$ & $0(0)$ \\
$1960 \mathrm{~s}$ & $3(4)$ & $38(51)$ & $0(0)$ & $58(78)$ & $1(1)$ \\
\hline
\end{tabular}

* Mean percent for all categories of film within each decade.

Considering first, single element transitions (i.e., a simple dissolve from one scene to another, or a simple fade down on one scene and fade up on another, with no inserted titles or shots within the transition), the data shows a heavy use of the fade in the 1930 s (approximately $46 \%$ of all single element transitions in the sample employed a fade), con- 
siderable use of the dissolve (approximately $44 \%$ of the single element transitions were a dissolve), and occasional use of the wipe (9\%). In the 1940s, the major figures shift significantly: $27 \%$ of the single element transitions employ a fade; $64 \%$ are dissolves; use of the wipe declines slightly to $5 \%$; and we encounter a few examples of straight cut transitions (3\%). In the 1950s, the trend continues. Fades drop to $13 \%$ of all single element transitions; dissolves account for $66 \%$; and the straight cut emerges with $21 \%$ of the transitions. By the 1960s, the fade is used in only $3 \%$ of the single element transitions; $38 \%$ are dissolves; the straight cut increases in usage to $58 \%$; and there was one case where a shift in focus (i.e., the scene goes out of focus, and then returns to a sharp focus revealing a new scene at a different point in time and space) signaled a transition.

There were no significant differences across film subjectscomedy, drama, etc. Variations from the general pattern by individual films are accounted for, primarily, by the number of "expressive" transitions in the film (this will be discussed later). Data on silent films of the 1920s was not included here because they depend heavily on multiple element transitions. (See Table II.)

TABLE II TRANSITION TIME

\begin{tabular}{lc}
\hline & *Seconds \\
\hline $1920 \mathrm{~s}$ & 6.7 \\
$1930 \mathrm{~s}$ & 4.8 \\
$1940 \mathrm{~s}$ & 3.1 \\
$1950 \mathrm{~s}$ & 1.8 \\
$1960 \mathrm{~s}$ & .4 \\
\hline
\end{tabular}

* Mean time for all transitions, single element and multiple element.

The length of time employed in completing a transition shows a similar trend diachronically, with a consistency among films within a period. Considering all transitions, single element and multiple element, the mean time for completing a transition declines steadily from the 1920 s through the 1950s. The sharp drop in mean time during the 1960 s reflects a sharp decrease in the use of multiple element transitions.

Within a film, variation in length of time to complete a transition is clearly patterned. If a filmmaker wishes to make a transition, but not attach "expressive" meaning (I will be using the term "expressive" to cover a variety of mood feelings the filmmaker wishes to imply, e.g., sadness, as well as dramaturgical meaning such as "this is an important transition"), he completes the transition within a time that is close to the mean time for that period. "Expressive" meaning is attached by employing the mean transition time of earlier films (which, it turns out, is always longer). For example, if the typical single element transition takes one second, and the filmmaker employs a 1.5 or 2 second transition, it will imply some "expressive" meaning. A viewer notices this as an "overlong" dissolve or "overlong" fade that accompanies an important transition in the film. For example, $A$ Man For All
Seasons (1966), uses "overlong" dissolves when there is a temporal-spatial transition at moments of heightened dramatic tension.

In some films of the 1920 s and 1930s it also appears to be the case that variations in the length of a transition served as an analogue for the amount of time that had passed or the distance that had been spanned. Thus a transition which took longer than normal implied that much time had passed, and a short transition implied that only a brief amount of time had passed. The use of this convention appears to diminish by the 1950s, 60s, and 70s. However, the passage of much time or any shift back in time (the flashback) is still typically characterized by an "overlong" transition. (See Table III.)

TABLE III

MULTIPLE vs. SINGLE ELEMENT TRANSITIONS

\begin{tabular}{lccccc}
\hline & \multicolumn{3}{c}{$\begin{array}{c}\text { Multiple } \\
* \%\end{array}$} & $\begin{array}{c}\text { Element } \\
(\mathrm{N})\end{array}$ & \multicolumn{3}{c}{$\begin{array}{c}\text { Single } \\
\%\end{array}$} & $\begin{array}{c}\text { Element } \\
(\mathrm{N})\end{array}$ \\
\hline $1920 \mathrm{~s}$ & 66 & $(83)$ & 33 & $(41)$ \\
$1930 \mathrm{~s}$ & 23 & $(35)$ & 77 & $(114)$ \\
$1940 \mathrm{~s}$ & 18 & $(33)$ & 82 & $(146)$ \\
$1950 \mathrm{~s}$ & 13 & $(20$ & 87 & $(138)$ \\
$1960 \mathrm{~s}$ & 3 & $(4)$ & 97 & $(134)$ \\
\hline
\end{tabular}

* Mean percent for all categories of film within each decade.

If we look at the number of multiple element transitions (i.e., where one or more shots are inserted within the transition itself) against the total number of transitions in a film, we find a marked dependence on multiple element transitions in the 1920s (66\%), a leveling off between 18-23\% from the 1930s through the 1950s, and a sharp drop to only $3 \%$ in the 1960 s.

The multiple element transition often serves two functions: it implies a transition in time and space, and it raises the information state of the audience. That is, while the filmmaker is stepping "outside" the film, to make a temporal-spatial transition, he will frequently use the occasion to tell us some detail about a character or the action that we could not or might not have inferred from the film. In the 1920s, this was accomplished predominantly through the use of titles: "Later, our hero waits anxiously for the letter to arrive." In The King of Kings (1927), the inserted title is sometimes a quote from the bible, so the moral message of the scene is rather explicitly reinforced. Such dependence on lexical information, in a medium (silent film) praised for the sophistication of its visual code is not often pointed out.

The function of multiple element transitions in the 1930 s was quite similar. However, the title insert was now replaced (often) by inserts of a newspaper headline, a note written by one of the characters, a program from a play one of the characters was about to attend, etc. For example, a scene dissolves to a newspaper headline- "Strike Vote Due Tomorrow"- which dissolves to a sub-headline- "Violence is Predicted"-which dissolves to a scene outside a factory with workers and police about to confront each other. By the 
1940s, we still see a few lexical inserts, but more and more, the inserts are symbolic visuals: a worn-out boot, a bottle that is nearly empty, a ship sinking. By the 1950s, inserts within multiple element transitions carry less information. It appears that the information state of the audience is raised merely by the use of a multiple element transition. The filmmaker does not have to insert an explicit visual to imply something about a character or the action. The structure itself implies "expressive" meaning. For example, in $A$ Hatful of Rain (1957), a multiple element transition occurs when Eva Marie Saint is going home to tell her husband (a drug addict) that she is through with him. The visual inserts within the transition are neither dramatic, nor are they necessary to give the viewer information about the transition. The presence of the multiple element transition form, rare both for the late 1950s and this film, serves to heighten the tension of the expected confrontation.

Thus, the symbolic encoding attached to a title in the 1920s, moved to a telegram or newspaper headline in the 1930 s, a visual symbol in the 1940s, and a visual structure in the 1950s. By the 1950s, audiences had learned to associate "expressive information" with multiple element transition structures, so a filmmaker, in drawing upon that structure, could imply expressive information without the explicit inserts that were necessary earlier.

By the 1960s, multiple element transitions decline sharply, and the non-temporal-spatial information that was encoded in titles, visuals, or the multiple element transition structure itself, moves, in part, to the shots immediately preceding or succeeding the transition-shots that are part of the ongoing film. For example, in the 1940s we might have a sequence in which we see a character in a room. This shot then dissolves to a pair of new boots, dissolving to a shot of a pair of worn out old boots, which then dissolves to a shot of the character later in life, old and run down. On the other hand, in the 1960s, a filmmaker might show us the same sequence of a character in a room but have the camera paining from his face to a pair of new boots in the corner of the room. There would then be a straight cut to a pair of old boots in a matching frame, and a pan back to the character, old and run down. Here, the meaning-laden insert within the multiple element transition of the 1940s, moves to the preand post-transition scenes. In this new position, the symbolic encoding must function at two levels. The "boots" must function as a proper element within the ongoing film, plus carry a special meaning by virtue of their proximity to a temporal-spatial transition. I believe we can draw a limited analogy, in terms of information state, with the theater. It's similar to the difference Goffman (1974:232-233) notes between an aside in a play spoken directly to the audience, which is outside the official information state of all the characters (except the characters speaking the line) and therefore only has meaning to the audience, and a line between two characters in the play which has one meaning for the characters and a second, special meaning to the audience because they have a different information state than the (official) information state of the characters in the play.

Thus far, we have been considering the nontemporal-spatial information imbedded in multiple element transition mechanisms. However, temporal and spatial in- formation follows a similar development. We find a title in the 1920 s saying, "Years later on their 25th anniversary"; a telegram in the 1930s, "Dear Mary ... Stop ... Happy 25th Anniversary ... Stop ... George"; a cake in the 1940s, with "Happy 25th Anniversary" spelled out in candles. By the 1950 s, the multiple element transition structure itself would likely carry the expressive information that much time had passed, and the explicit information that it is their 25th anniversary would probably not be conveyed within the transition, but revealed in the subsequent scene.

With the decline of multiple element transitions in the 1960s, and increased use of the straight cut to imply a temporal-spatial transition, we can ask, how do people know that a transition has occurred? What is the difference between a cut within a scene and a cut that signals a temporal-spatial transition? Just as the meaning-laden insert within multiple element transitions moved to the scenes before and after the transition, information signalling a temporal-spatial transition in the 1960 s often moves into the scenes before and after a cut. This is the kind of transition popularized in the TV series Mission Impossible: a camera zooms in on an ash tray; there is a cut to another ash tray; and the camera zooms out to another scene. Similarly, in Planet of the Apes (1968) the camera pans up to the sun; there is a cut to another shot of the sun from a slightly different angle; and the camera pans down to another scene at another point in time and space. In each of these instances, the ash tray or the camera movement functions within the ongoing scene, and implies a second meaning by virtue of the shared structural knowledge between filmmaker and audience that this pattern signals a temporal-spatial transition.

It should be noted that one can observe similar patterns of temporal and spatial transitions much earlier. However, in the past such patterns were accompanied by other structural information (e.g., a fade or dissolve) which implied the temporal-spatial transition. A straight cut does not imply a temporal-spatial transition in all contexts.

Finally, we may consider some of the patterned variations wtihin a given film that point toward the process of code change. If we look at the first element in a multiple element transition, and the first four single element transitions in a film, there is a tendency to use the convention patterns of earlier films. For example, in the 1940s, the general pattern shows $27 \%$ of the single element transitions are fades, and $64 \%$ dissolves. However, at the beginning of films in the 1940 s (i.e., the first four temporal-spatial transitions) the pattern is $42 \%$ fades and $54 \%$ dissolves. Similarly, the pattern at the beginning of a multiple element transition is $36 \%$ fades and $48 \%$ dissolves. In both of these situations, the pattern is closer to the convention of the 1930s. The filmmaker, at the beginning of his film or at the beginning of a complex, multiple element transition, relies to a greater degree on earlier conventions that are more likely to be understood by the widest possible audience. As his film progresses, his transition structure moves toward the mean for that period. Also, he may begin to experiment with new forms. In time, the mean transition mechanism of a period and filmmakers' experimentations become more deeply understood by wider audiences, which allows these transitions to be invoked at the beginning of a film to clearly establish a scene, at moments 
when there is a complex transition, and at moments of "expressive" meaning (there is a tendency to use the older convention mechanism for expressive meaning, just as we saw a tendency to use the earlier mean time for expressive transitions). This process would allow the code to evolve.

We can also see the process of code change in the way a filmmaker uses a new visual symbol as an insert in multiple element transitions, or an unconventional structural mechanism in a single element transition. During the 1930s and 1940s, there were a number of conventional inserts for multiple element transitions, e.g., a clock with rotating hands, a ship crossing the Atlantic, a train going around the bend, a flower blossoming, etc. If a filmmaker drew upon such a conventional symbol, he could expect that his audience would infer what he meant without additional information (other than the multiple element transition structure). The audience could refer the symbol to their knowledge of other films where that symbol had a particular meaning, and thereby know what he meant. However, the filmmaker was also free to create a unique symbol for his film. When he wanted to use a symbol of his own, he had to negotiate this new code item with his audience. He did this by initially using the symbol in a fuller context that told the audience what he meant, then repeating it later without the fuller context. For example, in Lloyd's of London (1936), a shot of a ship's bell is used throughout the film to imply a temporal-spatial transition. The first time the bell is used, we see its full context: it's an old ship's bell in a tavern. When news comes in, they ring the bell and post the news on a blackboard. Later, the bell is used without its full context to imply a transition in time and to fill in news. An audience can then refer the code item to its fuller context (which they experienced early in the film) and thus infer meaning just as they do by referring a conventional code item to the larger context they have learned from watching films. ${ }^{5}$

Similarly, if a filmmaker wishes to use an unconventional mechanism in single element transitions, he typically introduces it in a setting that clearly establishes how he is using it and what it means. Later, he can repeat the mechanism without this additional information. For example, in The Outsider (1962) a swish pan ${ }^{6}$ (i.e., where the camera pans across a scene very rapidly, causing the image to blur) is employed to signal a temporal-spatial transition. When the audience first sees this mechanism, it is clear from the context that a transition has taken place. Also, film viewers readily infer that expressive meaning has been attached to the swish pan-it suggests that a character is becoming confused and losing control of the situations in which he finds himself. Later in the film, the swish pan can be used to imply both a transition and expressive information about the character's loss of control over situations. Audiences refer subsequent experience with the transition mechanism to earlier experiences in the film, where they learned what it meant.

Some preliminary conclusions about a few of the explanations for temporal-spatial transition mechanisms suggested earlier can now be suggested. There appear to be no significant variations in transition patterns by category of fiction film. There are variations within a film that relate to the mood a filmmaker wants to imply. However, mood is implied by deviance from the convention at a given period (typically, toward the earlier convention), not in a code item such as fade, per se. Similarly, a film may vary from the conventions at a given period to identify with an earlier group of films, and align the audience's expectations with those earlier films. For example, a "grade B" western made in the 1960s, but following the typical story line of a 1940 western, may employ several multiple element transitions, inserts of newspaper headlines, etc. ${ }^{7}$ We have discovered no universal rules. In fact, our evidence points toward the conclusion that film structure (at least, regarding temporal and spatial transitions) is subject to constant renegotiation between filmmakers and their audiences.

It does appear that some mode borrowing occurred early in the history of film, via titles, the wipe, the fade, etc. and these structural mechanisms diminished in use as the film code evolved. ${ }^{8}$ This investigation provides no evidence about possible linguistic determination of the film code. Similarly, the study provides no evidence about technological influence on film structure. However, I would argue that while technology may introduce a new transition mechanism or create some incentive for an existing one, the change in code convention would have to be negotiated between filmmakers and their audience in a manner similar to other code changes.

Second, the investigation points to the evolution of a more symbolic visual code for temporal and spatial transitions. Filmmakers no longer have to "tell" their audiences that a temporal-spatial transition is taking place. We saw the use of titles in the 1920s evolve to visual objects with lexical information (i.e., the cake which spells out "Happy Anniversary"), which evolved to visual objects alone, and then to visual structure. All along, the code has become more efficient, in the sense of accomplishing the transition in less time, and we have seen the development of code items which serve dual functions, i.e., the visual object or camera movement which exists within the ongoing film scene and has a meaning in relation to that scene, while having a second meaning by virtue of its proximity to a temporal-spatial transition and its structural similarity to another object or camera movement in a subsequent scene. Thus, more of the meaning is encoded in structural relations and less in explicit linguistic or pictographic terms. This suggests that mass audiences have grown in their level of understanding the film code. That is, not only have they adapted to changes in transition mechanisms, but they have learned to perceive and understand code items of a more symbolic nature in considerably less time.

Third, investigation of this narrowly defined code appears to support Bateson's general position that communication codes are not static systems, but negotiated conventions. Focusing more specifically on film communication, I would modify Worth's position slightly (see earlier), and argue that a filmmaker must constantly refer what he proposes to do and the meaning he would attach to it, with what other films do at that time, what earlier films have done, and the set of expectations an audience will likely apply to his film. He must provide his audience not only with a code item that implies the meaning he desires, but he must give them sufficient information so they can refer the code item to the proper set of conventions and contexts in which this code item has the meaning he intends to communicate.

For example, if I see a film today that has a shot of 
calendar pages flipping off a wall (during a multiple element transition), and I know the film was made in the 1930s, I will likely infer that it is a perfectly reasonable transition; if something in the film suggests to me that it was produced in the 1950s, the calendar pages shot may seem incongruent; if something in the film suggests it was produced in the late 1960s, I might laugh-at the filmmaker if I felt he intended a non-expressive transition, and with him if I felt he intentionally used a convention from the 1930s for a comic effect. More generally, a filmmaker must communicate to his audience (through camera angles, lighting, sound, etc., as well as transition mechanisms) that he is adhering to the viewers' patterned expectations of code conventions for the period of time when the film was made; deviating from those conventions toward another set of conventions the audience knows (e.g., a modern gangster film about the 1930s may borrow certain code items from films of the 1930s); or deviating in a unique way, in which case he must not only teach them the new code item but provide the contextual references that will give the code item a meaning he intends when the audience encounters it again in the film or in some future film.

The study reported here suggests some directions for future research. (1) If we are correct in arguing that structural codes in film do not represent the surface manifestation of a universal deep visual structure, but negotiated conventions, it would follow that children must learn them. By studying how they acquire such knowledge and become competent viewers, we may learn a great deal about the codes themselves. (2) We have been able to show some features of one element in the film code, for American mass audiences, but we cannot assume that all audiences and filmmakers (i.e., in all cultures, or even sub-groups within one culture) share the same set of conventions. Rather, the boundaries for groups of filmmakers and audiences in different cultures, over time, and across other relevant dimensions, must be discovered. (3) The need for a great deal more systematic investigation of film structure is clearly indicated. Those of us who hope to conduct comparative studies of film and linguistic codes are forced to recognize that our present knowledge of film structure is inadequate for the task.

\section{NOTES}

${ }^{1}$ This is not to imply that an audience "naturally" understands this. A viewer must learn the conventions that allow him to infer no meaning. Further, this code issue has fascinating implications for crosscultural investigations. Montagu (1964:127) points out that pre
1950 Chinese films never speeded up such movement-the audience had not yet learned the conventions.

${ }^{2}$ The filmmaker does not intend to communicate any meaning from such a construction, and the audience does not know that the shots were filmed in different places, at different times.

${ }^{3}$ Three films for each category were selected, totaling nine films per decade. Films were chosen generally toward the middle of each decade, and an attempt was made to avoid both avant-garde and grade B films. Thus the sample was primarily standard Hollywood fare. There is no suggestion here that a decade is a natural unit for film structure. It is an arbitrary grouping that will, hopefully, give way to ,natural units (when they are discovered). Further, the small size and selectivity of the sample places some limitations on the generalizability of the findings. Clearly a large sample would be helpful to account for the widest possible range of films, grade B to avant-garde, feature length to TV commercial.

${ }^{4}$ There is a reliability problem in noting certain semantic features. Since I was the only coder, a mood feature like "sadness" is subject to the systematic bias of my observation. Therefore, all mood features and dramaturgical meaning like "this is an important transition" were placed in one broad category - "expressive."

${ }^{5}$ The same principle is true for sound symbols used in transitions. The structure of auditory transition mechanisms, generally, will be reported in a later paper.

${ }^{6}$ It should be noted that the swish pan was not unique at this point (it simply was not present in the sampled films), and has since become well understood by a wide audience through use in many television series during the 1960s.

${ }^{7}$ Also, a modern film about the 1930 s may employ the transition mechanisms common in films of the 1930s.

${ }^{8}$ Amos Vogel (personal communication) suggests that many of the transition patterns used in Hollywood features of the 1960 s and 1970 s were borrowed from earlier avant-garde films.

\section{REFERENCES CITED}

Arnheim, Rudolph

1957 Film as Art. Berkeley: University of California Press.

1966 Toward a Psychology of Art. Berkeley: University of California Press.

Balázs, Béla

1970 Theory of the Film. New York: Dover.

Bateson, Gregory

1969 Chapter 1. In The Natural History of an Interview. N. McQuown, Ed. Chicago: University of Chicago Press.

Goffman, Erving

1974 Frame Analysis: The Organization of Experience. New York: Harper and Row.

Gombrich, E. H.

1960 Art and Illusion. Princeton: Princeton University Press.

Montagu, Ivor

1964 Film World. Gretna, LA: Pelican Books.

Pudovkin, V.I.

1949 Film Techniques and Film Acting. New York: Lear. Worth, Sol

1975 Pictures Can't Say Ain't. Proceedings, First Annual International Conference in Semiotics. The Hague: Mouton (in press). 